\title{
Ambulatory Blood Pressure Monitoring of Patients with Heart Failure. A New Prognosis Marker
}

\author{
Manoel F. Canesin, Dante Giorgi, Múcio T. de Oliveira Jr., Maurício Wajngarten, Alfredo J. Mansur, \\ José Antonio F. Ramires, Antonio Carlos Pereira Barretto
}

São Paulo, SP - Londrina, PR - Brazil

Objective - To evaluate the relationship between 24-hour ambulatory arterial blood pressure monitoring and the prognosis of patients with advanced congestive heartfailure.

Methods - We studied 38 patients with NYHA functional class IV congestive heart failure, and analyzed left ventricular ejection fraction, diastolic diameter, and ambulatory blood pressure monitoring data.

Results - Twelve deaths occurred. Left ventricular ejection fraction $(35.2 \pm 7.3 \%)$ and diastolic diameter $(72.2 \pm 7.8 \mathrm{~mm})$ were not correlated with the survival. The mean 24-hour (SBP24), waking (SBPw), and sleeping (SBPs) systolic pressures of the living patients were higher than those of the deceased patients and were significant for predicting survival. Patients with mean SBP24, SBPv, and $S B P s \geq 105 \mathrm{mmHg}$ had longer survival $(p=0.002, p=0.01$ and $p=0.0007$, respectively). Patients with diastolic blood pressure sleep decrements (dip) and patients with mean blood pressure dip $\leq 6 \mathrm{mmHg}$ had longer survival ( $p=0.04$ and $p=0.01$, respectively). In the multivariate analysis, SBPs was the only variable with an odds ratio of 7.61 (CI: 1.56; 3704) $(p=0.01)$. Patients with mean $\mathrm{SBP}<105 \mathrm{mmHg}$ were $7.6 \mathrm{ti}$ mes more likely to die than those with $S B P \geq 105 \mathrm{mmHg}$

Conclusion - Ambulatory blood pressure monitoring appears to be a useful method for evaluating patients with congestive heart failure.

Key words: heart failure, prognosis, blood pressure ambulatory monitorization

Heart Institute of Hospital das Clínicas - FMUSP - São Paulo and University Hospital - HURNP/UEL - Londrina

Mailing address: Manoel F. Canesin - Rua Piauí, 701- 86020-320 - Londrina, PR Brazil - E-mail: mcanesin@ cardiol.br
Congestive heart failure is a complex syndrome with various clinical presentations each having different etiological and pathophysiological characteristics. The particular clinical variation is related to the great number of signs and symptoms, to the myocardial dysfunction, and to the neurohormonal activation that each patient experiences. With such variability, it is difficult to correctly establish the severity and prognosis of congestive heart failure.

Despite the study of the therapeutic value of medications, large clinical trials have identified hemodynamic, neurohormonal, immunological, and inflammatory alterations ${ }^{1}$ that have distinct prognostic value according to the evolutionary stage of the heart failure, characterizing this syndrome as a complex disease.

Among the countless parameters used to evaluate the patients' evolution, 2 prognostic indexes are considered of great importance: New York Heart Association (NYHA) functional class ${ }^{2}$ and left ventricular ejection fraction ${ }^{3}$. Due to the ease of attainment and their discriminatory powers, these indexes are currently the most frequently used for stratifying patients' risk during different evolutionary phases of the disease. Often, however, due to the subjectivity of functional class evaluation or to failure to evaluate left ventricular ejection fraction, it is not possible to determine the evolutionary difference of advanced congestive heart failure patients with these indexes. Prognostic indexes, such as systolic volume and final left ventricle diastolic pressure $^{4}$, are also used as criteria for indication of heart transplant in patients in more advanced stages of the disease. However, often they do not allow differentiation of patients with a worse evolution. Possibly because these indexes are evaluated only once, difficulties still exist in determining the correct prognosis of patients with advanced congestive heart failure. In some studies, left ventricular ejection fraction did not correlate with survival time in advanced congestive heart failure patients ${ }^{5}$ and, probably, the lack of correlation may be explained by the fact that these methods do not assess myocardial reserve. Factors like the hemodynamic profile, blood pressure, and heart rate may influence the 
ventricular ejection fraction of patients with advanced congestive heart failure ${ }^{6}$. Along the same line of reasoning, other factors, such as clinical signs and symptoms, biochemical, hematological, and neurohormonal parameters evaluated at rest, do not predict a better prognosis for advanced congestive heart failure.

Some studies have already analyzed the role of dynamic evaluation in patients with advanced congestive heart failure. Maximum oxygen consumption by ergo-spirometric testing ${ }^{7}$, measurement of maximum heart rate on effort ${ }^{3}, 6$ minute walk testing ${ }^{8}$, stress echocardiography ${ }^{9}$, and maximum oxygen consumption ${ }^{7}$ are being used to evaluate these patients' prognoses. Other dynamic variables carried out for longer periods may be used to evaluate congestive heart failure patients. Alterations in the blood pressure levels and their variability over 24 hours, evaluated by 24 -hour ambulatory blood pressure monitoring ${ }^{10}$, have not yet provided criteria for determining prognostic indexes, especially in advanced congestive heart failure patients.

Due to the difficulty of determining the prognosis of patients with advanced congestive heart failure with greater precision through rest methods, new methods of evaluation, applicable to most patients, are still needed. The assessment of methods for dynamic evaluation may provide a better definition of prognosis and of treatment for these patients. We used ambulatory blood pressure monitoring as a method of dynamic evaluation over 24 hours to assess blood pressure behavior in a group of patients with advanced congestive heart failure, and we tried to establish a correlation between ambulatory blood pressure monitoring variables and these patients' prognoses.

\section{Methods}

Forty-three patients admitted to the emergency room with a clinical diagnosis of congestive heart failure functional class IV (NYHA) were prospectively studied. At the time of admission, they did not have primary valve diseases capable of correction, congenital heart disease, features of acute coronary ischemia, or surgery for coronary revascularization for at least 6 months, or carditis, endocarditis, and pericarditis.

All patients received classical treatment for severe, chronic arrhythmic congestive heart failure, which included rest, a low-salt diet, and restriction of water intake to 800 to $1000 \mathrm{~mL} /$ day. Medication therapy included digitalis administered orally to 39 patients, $0.25 \mathrm{mg} /$ day of digoxin, and in 4 patients $0.100 \mathrm{mg} /$ day of digoxin. Diuretic therapy included use of snare diuretics (furosemide, 40 to $240 \mathrm{mg} /$ day) adjusted according to patients' diuretic response. Thiazide diuretics (hydrochlorothiazide, 25 or $50 \mathrm{mg} /$ day) were prescribed to 11 patients and spironolactone to four $(25 \mathrm{or} 50 \mathrm{mg} /$ day). Angiotensin-converting enzyme inhibitors used were captopril (50 to $100 \mathrm{mg} /$ day) in 33 patients and enalapril (5 to $20 \mathrm{mg} /$ day) in 4 patients. In 6 instances of contraindication or undesirable side effects to angiotensin-converting enzyme inhibitors, hydralazine ( 75 or $100 \mathrm{mg} /$ day) was used in conjunction with isosorbide dinitrate (60 to $120 \mathrm{mg} /$ day) as a vasodilator option. Nine patients had signs of low heart debt associated with persistent congestive manifestations, it being necessary to use inotropic support with dobutamine, 5 to $10 \mathrm{mg} / \mathrm{kg} / \mathrm{min}$, iv, maintaining the therapeutic schedule and removing dobutamine after clinical stabilization. After clinical compensation of the acute phase, patients were informed of the study, and were asked to give written consent to participate.

After hemodynamic stabilization and reduction in congestive status, all 43 patients were placed on a 24-hour ambulatory blood pressure monitoring and echocardiographic evaluation of left ventricular ejection fraction and left ventricular diastolic diameter. After hospital discharge, patients were followed up as outpatients. All patients were contacted, within a minimum observation period of 6 months (28 weeks). The date of death, when it occurred, was confirmed by the death certificate or by first degree relatives' information. None of the patients underwent heart transplantation or any other surgical procedure during the study.

Patients were predominantly male, 29(67\%). Mean age of the group was $53.5 \pm 13.0$ years, with the youngest being 21 years old and the oldest being 76. Most of the patients, 37 $(86 \%)$, were over 40 years old, and only $6(14 \%)$ were between 21 and 39 years of age. The onset of congestive heart failure symptoms was reported on average 41 months before inclusion in the study, varying from 0.5 to 240 months of evolution. Most patients reported the first signs of the disease between 1 and 5 years before hospitalization. Hypertensive cardiomyopathy was the cause of congestive heart failure in 16(37\%) patients; idiopathic dilated cardiomyopathy in 14(32\%) patients; chagasic cardiomyopathy in $8(18 \%)$ patients, and ischemic cardiomyopathy in 5 patients $(13 \%)$.

After congestive heart failure compensation was established, patients underwent ambulatory blood pressure monitoring, by the oscillometric principle using the 90207 SpaceLabs monitor (SpaceLabs Inc., WA, USA). Data analysis was performed using 90121 SpaceLabs report managing software installed in a personal computer. Five patients either did not undergo the examination or correct data interpretation was not possible. Two refused monitor installation or its maintenance; 1 had high-response atrial fibrillation, 1 had atrial tachycardia, and 1 patient had ventricular extrasystoles and non-sustained ventricular tachycardia episodes difficult to control.

The monitor's cuff was put in place by a trained nurse in the morning on the patient's nondominant arm and removed at the same time the following morning. The patients received a diary to record any unexpected events, time of serum onset, and wake-up time, and were instructed to relax the cuffed arm at the time of insufflation. They were also instructed to take at least 1 walk during the recording period. Meals were served at the same time to all patients. The monitor was programmed to record blood pressure every 15 minutes within a 24-hour period. Minimum criteria to validate the recording were more than 60 successful readings from a total of 95 scheduled, with at least 2 efficient readings per hour. 
Analysis of data collected from 38 patients, adjusted to the waking and sleeping periods according to patients' reports: included: mean 24-hour systolic blood pressure (SBP24), mean 24-hour diastolic blood pressure (DBP24h), mean 24-hour mean blood pressure (MBP24), mean waking systolic blood pressure (SBPw), mean waking diastolic blood pressure (DBPw), mean waking blood pressure (MBPw), mean sleeping systolic blood pressure (SBPs), mean sleeping diastolic blood pressure (DBPs), and mean blood pressure during sleep (MBPs). Blood pressure variability for the period and the decrements in SBPw and SBPs (dipSBP), DBPw and DBPs (dipDBP), MBPw and MBPs (dipMBP) were also calculated.

Initially, all variables were descriptively analyzed. Continuum variables were analyzed by observation of minimum and maximum values and by calculation of means and standard deviations. For classificatory variables, absolute and relative frequencies were calculated. Correlation between variables was performed with Pearson's correlation coeficient ${ }^{11}$. Association between each variable measured and death was analyzed with Student's $t$ test ${ }^{11}$ for parametric variables and the chi-square test or Fisher's exact test for classificatory variables. To evaluate the association of variables together with death, a multiple logistic regression with a process of stepwise selection was used ${ }^{11}$. To study the patient's time of survival, the Kaplan-Meier survival curves and Cox's regression model for analysis of joint variables were used. The level of significance was set at $5 \%$.

\section{Results}

Echocardiographic measurement of left ventricle diastolic diameter varied from 56 to $92 \mathrm{~mm}$, with a mean \pm S.D. of $72.2 \pm 7.8 \mathrm{~mm}$. Thirty patients $(70 \%)$ had an average diameter of $70 \mathrm{~mm}$ or more. Left ventricular ejection fraction determined by echocardiography varied from 18 to $52 \%$, mean $35.2 \pm 7.3 \%$. Values $\leq 35 \%$ were observed in $22(51 \%)$ patients.

Means and standard deviations of patients' blood pressure measurements throughout the period were: SBP24, $108.2 \pm 13.4 \mathrm{mmHg}$; DBP24, 72.2 $\pm 8.1 \mathrm{mmHg}$; and MBP24, $84.9 \pm 8.6 \mathrm{mmHg}$. Means and standard deviations of blood pressure during waking periods (SBPw, 109.0 $\pm 13 \mathrm{mmHg}$; DBPw, $72.2 \pm 7.9 \mathrm{mmHg}$; and MBPw, $85.5 \pm 8.4 \mathrm{mmHg}$ ) were higher when compared to with pressure averages and standard deviations throughout the sleeping period [SBPs, $106.1 \pm 15.5 \mathrm{mmHg}$;DBPs, $70.5 \pm 9.6 \mathrm{mmHg} ; \mathrm{MBPs}, 83.3 \pm 10.3 \mathrm{~mm}$ $\mathrm{Hg},(\mathrm{p}=0.004, \mathrm{p}=0.005$, and $\mathrm{p}=0.009$, respectively) $]$. Means and standard deviations of the night decrements in patients throughout the period were: dipSBP, $2.8 \pm 5.6 \mathrm{mmHg}$; dipDBP, $2.2 \pm 4.6 \mathrm{mmHg}$; and dipMBP, $2.1 \pm 4.6 \mathrm{mmHg}$ with a drop of $2.6 \%, 3.0 \%$, and $2.5 \%$ during the sleeping period compared with that in the respective waking period.

A statistical correlation existed between the measures of blood pressure and left ventricular ejection fraction or with left ventricular diastolic diameter, evaluated by 24 -hour ambulatory blood pressure monitoring (Table I). However, no correlation existed between night decrements and echocardiographically evaluated measures.

After a period of at least 28 weeks (6 months), $12(31 \%)$ deaths, $11(92 \%)$ men and one woman $(8 \%)$, were reported by phone contact, telegram, or verification of medical charts. Patients underwent an average follow-up of 35 weeks ( 7.5 months), varying from 7 to 76 weeks. The 12 deaths were attributable to cardiopathy, with $9(75 \%)$ deaths in the hospital due to the disease's evolvement and 3 (25\%) sudden deaths at home, reported by relatives.

Mean and standard deviation of left ventricular ejection fraction ( $37 \pm 7 \%)$ of the live patients (32 patients) were higher than those $(32 \pm 6 \%)$ before death in patients (12 patients) who died ( $\mathrm{p}=0.02$ ). We also observed a tendency toward smaller left ventricular diastolic diameter $(7.1 \pm 0.8 \%)$ in the live patients compared with that in the deceased ones $(7.6 \pm 0.6 \%)(p=0.05)$. The Kaplan-Meier and log-rank nonparametric analyses indicated that both left ventricular ejection fraction and diastolic diameter were not significant predictors of the survival time of the patients under study.

Means and standard deviations of the average blood pressure of the live patients (26 patients) (SBP24, 111.5 \pm $13.1 \mathrm{mmHg}$; SBPv, $111.9 \pm 12.9 \mathrm{mmHg}$; and SBPs, $110.5 \pm$ $14.4 \mathrm{mmHg}$ ) were higher than those of the deceased patients (12 patients) before death [SBP24, 100 $\pm 11 \mathrm{mmHg}$; SBPv, $101.6 \pm 10.4 \mathrm{mmHg}$; and SBPs, $95.4 \pm 13.1 \mathrm{mmHg}(\mathrm{p}=0.01$, $\mathrm{p}=0.02$, and $\mathrm{p}=0.005$, respectively)]. Figure 1 shows the comparison between the 24-hour blood pressure curves of living and deceased patients. The mean and standard deviation of the live patients' MBPs $(85.6 \pm 9.2 \mathrm{mmHg})$ was higher than those of the patients who died $(77.6 \pm 11.0 \mathrm{mmHg})$ $(\mathrm{p}=0.02), \operatorname{dipSBP}(1.4 \pm 5.7 \mathrm{mmHg}), \operatorname{dipDBP}(1.1 \pm 4.8 \mathrm{mmHg})$, and dipMBP $(1.0 \pm 4.9 \mathrm{mmHg})$ of the live patients were lower than the dipSBP $(6.1 \pm 4.0 \mathrm{mmHg})$, dipDBP $(4.8 \pm 3.2 \mathrm{mmHg})$, and dipMBP $(5.0 \pm 3.3 \mathrm{mmHg})$ of the deceased patients ( $p=0.01, p=0.02$, and $p=0.01$, respectively) (Fig. 2). In the curves of mean systolic blood pressure measured hourly, the lower dipSBP in the live patients is noticeable. KaplanMeier and log-rank tests of nonparametric analysis revealed that SBP24, SBPw, SBPs, and MBPs were significant for the estimation of survival time of the study patients. Analysis of these parameters as classificatory variables revealed differences in survival time. The groups of patients with SBP24 $(\mathrm{n}=22), \mathrm{SBPw}(\mathrm{n}=23)$, and SBPs $(\mathrm{n}=23) \geq 105 \mathrm{mmHg}$ had a longer survival time than patients with SBP24 ( $\mathrm{n}=16), \mathrm{SBP}$

\begin{tabular}{|lllll|}
\hline \multicolumn{5}{|c|}{$\begin{array}{c}\text { Table I - Correlation between 24-hour, waking and sleeping } \\
\text { systolic blood pressure (SBP24, SBPw, SBPs) with left ventricular } \\
\text { ejection fraction (LVEF) and diastolic diameter (LVDD) } \\
\text { correlation coefficient) }\end{array}$} \\
\hline & \multicolumn{5}{c}{ (PVearson's } \\
Parameter & $\mathrm{r}$ & $\mathrm{P}$ & $\mathrm{r}$ & $\mathrm{p}$ \\
\hline SBP24 & 0.3500 & 0.0300 & -0.3772 & 0.0200 \\
SBPw & 0.3355 & 0.0300 & -0.3573 & 0.0200 \\
SBPs & 0.3719 & 0.0200 & -0.4096 & 0.0100 \\
\hline
\end{tabular}


$(\mathrm{n}=15)$, and $\operatorname{SBPs}(\mathrm{n}=15)<105 \mathrm{mmHg}(\mathrm{p}=0.002, \mathrm{p}=0.01$, and $\mathrm{p}=0.0007$, respectively) (Figs. 3 and 4 ). The group of patients with MBPs $(n=23) \geq 80 \mathrm{mmHg}$, when compared with patients with MBPs $(\mathrm{n}=15)<80 \mathrm{mmHg}(\mathrm{p}=0.0003)$ also had a longer survival time. The Kaplan-Meier and log-rank test for nonparametric analysis also revealed that dipSBP, dipDBP, and dipMBP were significant for estimation of survival time of the study patients. Analysis of these parameters as classificatory variables revealed the tendency toward differences in the longer survival time in the group of patients with dipSBP $\leq 6 \mathrm{mmHg}(\mathrm{n}=28)$, compared with that in patients with dipSBP $>6 \mathrm{mmHg}(\mathrm{n}=10)(\mathrm{p}=0.06)$. Patients with dipDBP $\leq 5 \mathrm{mmHg}$ and patients with dipMBP $\leq 6 \mathrm{mmHg}$ had longer survival times when compared with patients with $\operatorname{dipDBP}>5 \mathrm{mmHg}(\mathrm{p}=0.04)$ and dipMBP $>6 \mathrm{mmHg}(\mathrm{p}=0.01)$ (Fig. 5). The other blood pressure variables were significant as predictors of survival time in the patients studied according to the nonparametric analysis with Kaplan-Meier and log-rank tests. In the multivariate analysis, the variables with higher statistical power $(\mathrm{p}<0.10)$ were selected for the univariate analysis: age, sex, etiology, left ventricle ejection fraction, left ventricle diastolic diameter, SBPw and SBPs; SBPs was the only variable selected with an odds ratio of 7.61 (IC: $1.56 ; 37.04)(\mathrm{p}=0.01)$. Patients who had mean sleep systolic blood pressures $<105 \mathrm{mmHg}$ were 7.6 times more likely to die than patients with means $\geq 105 \mathrm{mmHg}$. In the Cox's regression model multivariate analysis, the variables associated with survival time were SBP24 ( $\mathrm{p}=0.07)$, SBPs $(\mathrm{p}=0.002), \mathrm{SBPw}(\mathrm{p}=0.01)$, and MBPs $(\mathrm{p}=0.01)$.

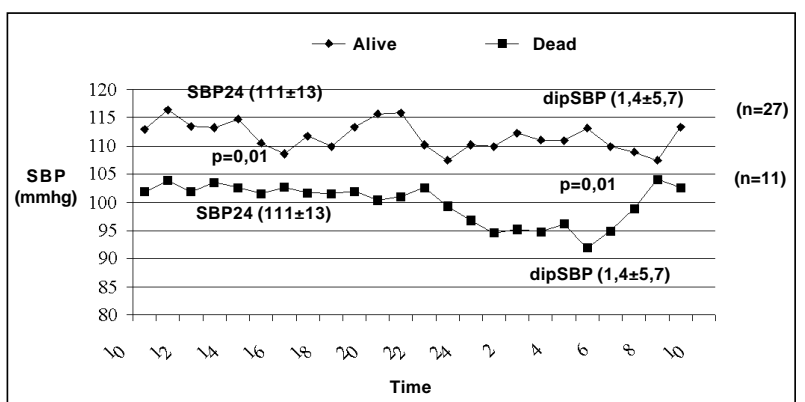

Fig. 1 - Comparative time-course of 24-hour systolic blood pressure (SBP) in $\mathrm{mm} \mathrm{Hg}$ between living $(\mathrm{n}=27)$ and deceased $(\mathrm{n}=11)$ patients in the sample.

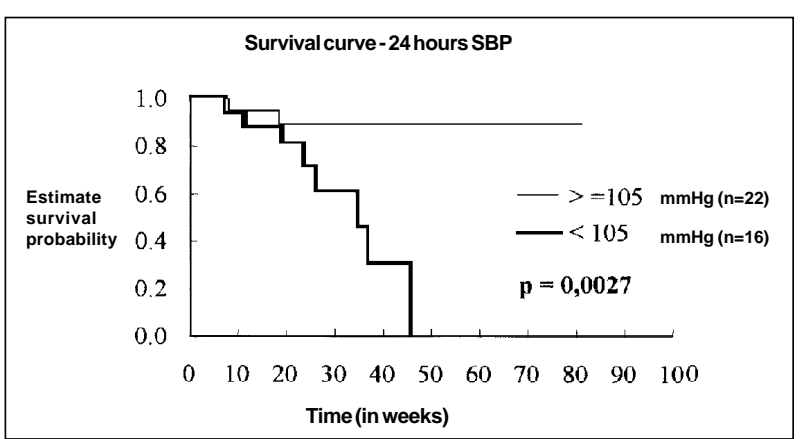

Fig. 2 - Survival curves of patients with mean 24-hour systolic blood pressure $(\mathrm{SBP} 24) \geq 105 \mathrm{mmHg}(\mathrm{n}=22)$ and of patients with SBP2 $4<105 \mathrm{mmHg}$ $(n=16)$.

\section{Discussion}

The purpose of the present study was to examine whether 24-hour dynamic evaluation through ambulatory blood pressure monitoring adds advantages to the study of patients with advanced congestive failure.

Several methods of direct evaluation of myocardial danger ay help in the prognostic evaluation $\mathrm{f}$ patients with advanced congestive heart failure. Among them are echocardiography; nuclear medicine techniques, such as radioisotopic ventriculography and myocardial perfusion scintigraphy; cardiac catheterization, and nuclear magnetic resonance. All these methods have advantages and limitations for myocardial anatomical-functional and prognostic evaluation of patients with advanced congestive heart failure. Analysis of myocardial impairment thecho-Dopplercardiography is one of the most used because it is a noninvasive examination, inexpensive, and easy to perform. Left ventricular ejection fraction and diastolic diameter used in the present sample are variables routinely used in the anatomical-functional and prognostic evaluation of patients with congestive heart failure, but nonetheless, are subject to criticism ${ }^{12-16}$. In clinical experience, it is evident that patients with larger ventricular dilation and smaller ejection fraction have a worse prognosis; however, it is not uncommon for patients with important compromise to have irrelevant clinical repercussion over a long evolutionary period. Although in some studies, such as ours,

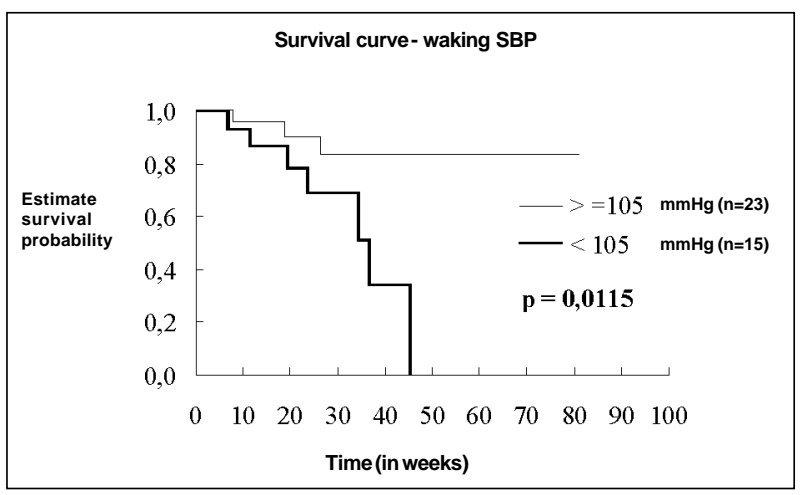

Fig. 3 - Survival curves of patients with mean waking systolic blood pressure $(\mathrm{SBPw}) \geq 105 \mathrm{mmHg}(\mathrm{n}=23)$ and of patients with $\mathrm{SBPw}<105 \mathrm{mmHg}$ $(n=15)$.

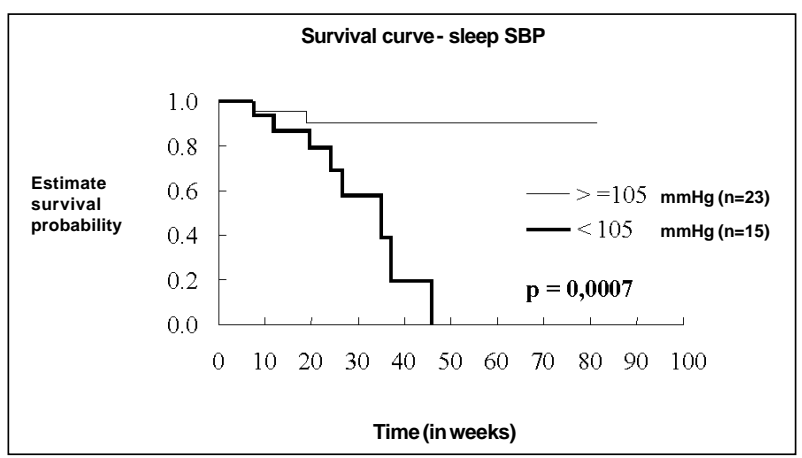

Fig. 4 - Survival curves of patients with mean sleep systolic blood pressure (SBPs) $\geq 105 \mathrm{mmHg}(\mathrm{n}=23)$ and of patients with SBPs $<105 \mathrm{mmHg}(\mathrm{n}=15)$. 


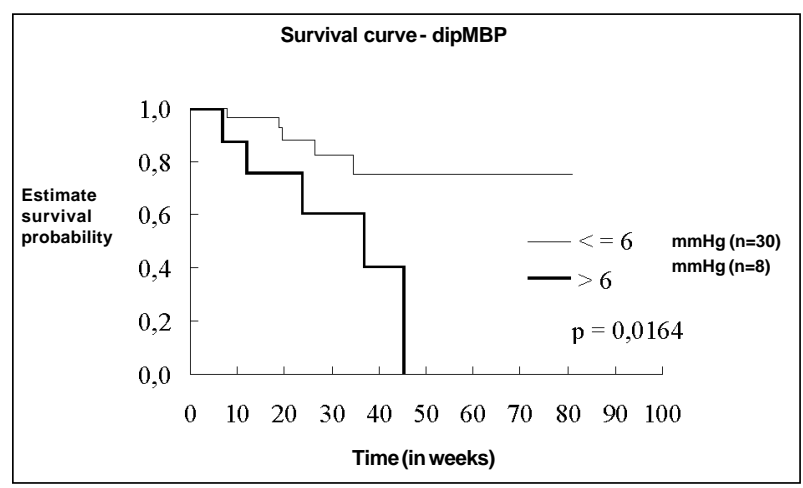

Fig. 5 - Survival curves of patients with mean blood pressure night decrements $(\operatorname{dipMBP}) \leq 6 \mathrm{mmHg}(\mathrm{n}=30)$ and of patients with dipMBP $>6 \mathrm{mmHg}(\mathrm{n}=8)$.

left ventricle ejection fraction did not show a correlation with the prognosis of patients with severe congestive heart failure ${ }^{17}$, it is important to emphasize that, in other studies, it was characterized as a variable, identifying patients with distinct evolutionary potentials ${ }^{15,17,18}$. The studies report that ventricular function indexes less influenced by loading conditions, such as the relation between final systolic pressure (or stress)/ final systolic volume (or diameter), and the relation between ejection fraction (or shortening percentage)/wall final systolic stress, are better mortality predictors than the classical indexes of ejection phase, such as ejection fraction, percentage of shortening of myocardial fiber, and velocity of circumferencial shortening ${ }^{20}$. Because results are not always homogenous and a search continues for more accurate methods of prognostic echocardiographic evaluation, dynamic evaluation through stress echocardiography is being used as a better complementary technique. Another form of prognostic evaluation for these patients is nuclear medicine; however, as occurs in echocardiography, factors like hemodynamic volume and blood pressure may influence ventricular ejection fraction of patients with advanced congestive heart failure who undergo radioisotopic ventriculography ${ }^{6}$.

Currently, several studies are underway that correlate ambulatory blood pressure monitoring with lesions in target organs in hypertensive patients. These studies used left ventricular hypertrophy ${ }^{20,21}$, microalbuminuria ${ }^{22}$, retina alterations, and cerebrovascular disease ${ }^{23}$ as variables. However, few studies used ambulatory blood pressure monitoring to investigate congestive heart failure ${ }^{10,24,25}$.

Average measurements of systolic and diastolic, 24hour, waking and sleeping blood pressures in patients with functional class IV congestive heart failure in the present sample were not higher than the normal patterns of ambulatory blood pressure monitoring. Note that clinically standardized normal values of isolated blood pressure measurements are not appropriate for ambulatory blood pressure monitoring. Recent population studies have proposed that maximum values of systolic and diastolic blood pressure should be between 119 to $126 / 75$ to $80 \mathrm{mmHg}^{26}$. With regard to ambulatory blood pressure monitoring values of congestive heart failure patients, Giles et al ${ }^{10}$ studied 30 patients with congestive heart failure, functional class II to IV with a nonhypertensive cause, and found higher mean 24-hour values of systolic $(130.8 \mathrm{mmHg})$ and diastolic $(76.5 \mathrm{mmHg})$ pressure, compared to with those in the present sample, which were between 108.2 and $72.2 \mathrm{mmHg}$, respectively. However, Borne et al ${ }^{24}$ studied 29 patients with congestive heart failure, functional class III and IV, excluding patients with hypertensive cardiomyopathy, and found lower systolic, diastolic, 24-hour, waking, and sleeping pressure values than those of our sample. These findings prove that other factors, such as degree of ventricular dysfunction, neurohormonal alterations, and the etiology of cardiomyopathy of patients with congestive heart failure, may lead to alterations in pressure levels, evaluated by ambulatory blood pressure monitoring of these patients.

In the analysis of blood pressure variability in the patients studied, means of decrements of systolic, diastolic, and sleeping average blood pressure were reduced, and a drop occurred during sleep of $2.6 \%, 3.0 \%$, and $2.5 \%$ as compared to with the respective measurements during waking time. The importance of blood pressure decrements during sleep is still controversial in healthy people and subjects with cardiovascular diseases. Verdecchia et al ${ }^{27}$ reported that hypertensive subjects not having blood pressure sleep decrements above $10 \%$ of the waking period had a more hypertrophied left ventricle when compared with patients with decrements during the sleeping period. Evaluating normotensive subjects ${ }^{28}$, these same authors did not find a difference in left ventricular mass between subjects with or without blood pressure decrements during sleep.

Regarding congestive heart failure, Borne et al ${ }^{24}$ found a smaller blood pressure and heart rate sleep decrement in patients with congestive heart failure, functional classes III and IV, as compared with a healthy population. The following hypothesis may explain the reduction in blood pressure sleep decrement in congestive heart failure patients. Nocturnal rest results in dispersion of retained liquids, leading to a concomitant increase in central venous pressure. In healthy people, the cardiopulmonary baroreflex inhibits sympathetic activation and helps decrease blood pressure. Some studies suggest a compromised congestive heart failure baroreflex may cause a reduction in blood pressure sleep decrement ${ }^{29-32}$. The finding may also be related to the increased sympathetic stimulation observed in congestive heart failure ${ }^{32}$. During the waking period, physical activity produces increased stimulation of the sympathetic nervous system, both in healthy subjects and in congestive heart failure patients. During sleep, this sympathetic activity, which remains stimulated in congestive heart failure patients, is inhibited with rest in healthy subjects. This fact possibly justifies also the lack of a decrement in blood pressure during sleep in congestive heart failure patients when compared with that in healthy subjects ${ }^{25}$.

Previous studies evaluated blood pressure variability in hypertensive patients through other parameters, such as standard deviation and the variation coefficient of blood pressure measurements ${ }^{33}$. Some studies in hypertensive patients showed that 24 -hour blood pressure variations were 
correlated with lesions in target organs ${ }^{34}$, but other studies did not show such a correlation ${ }^{35}$. In advanced congestive heart failure patients, these variables are subject to major alterations due to the influence of several pathophysiological factors of this phase of the disease, limiting data interpretation ${ }^{36}$.

Analysis of left ventricle ejection fraction and diastolic diameter showed, respectively, a positive and negative correlation with the following variables of blood pressure measurements: SBP24, SBPw, and SBPs. Left ventricular ejection fraction and diastolic diameter did not show a correlation with measurements of systolic, diastolic, and average blood pressure sleep decrement. These results demonstrated that left ventricular ejection fraction and diastolic diameter measured with echocardiography also have a correlation with systolic pressure levels evaluated by 24-hour ambulatory blood pressure monitoring. Caruana et al ${ }^{36}$ did not observe a correlation between SBP24, SBPw, and SBPs with left ventricular ejection fraction measured by ventriculography in patients with congestive heart failure, functional class II to IV; however, they showed a positive correlation of SBP24h standard deviation and blood pressure sleep decrement with left ventricular ejection fraction. In a study using functional class II to IV patients, Giles et al ${ }^{10}$ observed a negative correlation between systolic pressure absolute amplitude and neurohormonal indexes that are markers of congestive heart failure. Canesin et al ${ }^{25}$ observed a direct correlation between 24-hour systolic pressure measured by ambulatory blood pressure monitoring and the quality of life of patients with advanced congestive heart failure. Different findings of correlations between measures of blood pressure and its variability with left ventricular ejection fraction are probably due to the heterogeneous characteristics in the disease's evolutionary level, etiology, and even the presence of associated diseases in patients with congestive heart failure, which often hinder the selection of patients for such analyses.

Measurements of blood pressure may be correlated with the prognosis of congestive heart failure patients. Previous studies correlated blood pressure with survival time of patients with congestive heart failure. Franciosa et al ${ }^{37}$ reported that the isolated blood pressure measurement has a prognostic importance in patients with congestive heart failure. Other authors ${ }^{38}$ also demonstrated that higher values of isolated measurements of average blood pressure in patients with congestive heart failure, functional class II to IV, of different etiologies, are correlated with a better prognosis. Ghali et al ${ }^{39}$ demonstrated the prognostic role of isolated blood pressure measurement in congestive heart failure patients, where patients with smaller ventricular functions and higher systolic and diastolic blood pressures have longer survival time. These authors also observed that the same did not occur in congestive heart failure patients with better ventricular function, demonstrating that measurements of blood pressure in different populations of congestive heart failure patients may have different prognostic implications. From these studies of isolated blood pressure measurements began the analysis of continuous 24-hour dynamic evaluation of blood pressure.

In congestive heart failure patients, the use of ambulatory blood pressure monitoring is still limited. Using it in 29 patients with congestive heart failure, functional class III and IV, Borne et $\mathrm{al}^{24}$ found SBP24h, SBPw, and SBPs values lower than in the control group. In the present study, univariate analysis revealed that the patients who had higher 24hour, waking, sleeping, and averaged sleeping systolic blood pressure had lower mortality. In the analysis of survival time by Kaplan-Meier and log-rank tests, these same blood pressure variables were shown to be significant, demonstrating longer survival times in patients who had higher pressure levels. The classificatory analysis of blood pressure values showed that patients with mean values $\geq 105 \mathrm{mmHg}$ of SBP24h, SBPw, SBPs, and values $\geq 80 \mathrm{mmHg}$ of MBPs had longer survival times when compared with patients with lower values. Measurements of blood pressure variability evaluated by decrements during the sleep period also showed prognostic importance. The results demonstrated that deceased patients studied exhibited higher dipSBP, dip DBP, and dipMBP before death. Analysis of survival time by Kaplan-Meier and log-rank tests demonstrated, for the first time, that these variables have predictive value for survival time in patients with advanced congestive heart failure. Classificatory analysis showed a tendency toward a longer survival time in patients with dipSBP $\leq 6 \mathrm{mmHg}$ and a longer survival time in patients with dipDBP $\leq 5 \mathrm{mmHg}$ and dipMBP $\leq 6 \mathrm{mmHg}$. In Borne et al's ${ }^{24}$ study, using ambulatory blood pressure monitoring in congestive heart failure patients, functional class III and IV, of a nonhypertensive cause, systolic pressure circadian amplitude variations were correlated with the degree of ventricular dysfunction calibrated by cardiac index; however, their prognostic value was not evaluated. The analysis of ambulatory blood pressure monitoring of patients with congestive heart failure functional class IV of different etiologies in the present sample, revealed that patients with larger systolic blood pressure sleep decrements had shorter survival times. This finding is contrary to what has been previously described for hypertensive patients when those without night decrements had greater impairment of target organs. In congestive heart failure, Borne et $\mathrm{al}^{24}$ found smaller blood pressure sleep decrements and heart rates in these patients as compared with those in healthy. A possible explanation for a higher systolic blood pressure sleep decrement in the patients who died in our sample is the worse autonomic disturbance and to other still unknown pathophysiological factors of patients with advanced congestive heart failure.

In the univariate analysis, variables selected for predictive values of survival time were: age, sex, etiology, left ventricular ejection fraction, left ventricular diastolic diameter, SBPw, and SBPs. Using this model for multivariate analysis, the variable selected was average sleeping systolic blood pressure (SBPs), also revealing that patients who exhibit an SBPs $<105 \mathrm{mmHg}$ are 7.6 times more likely to die than patients with SBPs $\geq 105 \mathrm{mmHg}$. In the model of multivariate analysis by the Cox regression analysis, variables that were predictive for survival time were also SBP24h, SBPs, SBPw, and MBPs. These findings demonstrate the prognostic importance of systolic and average blood pressure in this group of patients with advanced congestive heart failure, 
particularly, SBPs, which was more closely correlated with survival in these patients than indexes of direct myocardial measurements, such as left ventricular diastolic diameter and ejection fraction.

These data demonstrate that measurement of systolic blood pressure with 24-hour ambulatory blood pressure monitoring in patients with advanced congestive heart failure may better represent the integration of the neurohormonal system, cardiac function, and peripheral vasculature than indexes that directly evaluate the myocardium, such as left ventricular ejection fraction and diastolic diameter. In general, we observed that lower systolic blood pressures and larger night decrements of blood pressure evaluated by 24- hour ambulatory blood pressure monitoring were predictors of higher mortality. Comparative analysis of survival plots suggests that parameters of systolic blood pressures and night decrements of systolic, diastolic, and average blood pressures obtained by ambulatory monitoring were predictors of mortality, which did not happen with left ventricular ejection fraction and diastolic diameter in this group of patients. These data seem to confirm the hypothesis that dynamic evaluation with ambulatory blood pressure monitoring is superior to that carried out at rest and that it may be a method of great usefulness for the evaluation of patients with advanced congestive heart failure, especially for establishing priority for heart transplantation.

\section{References}

1. Mann DL, Young JB. Basic mechanisms in congestive heart failure: recognizing the role of proinflammatory cytokines. Chest 1994; 105: 897-904

2. The Consensus Trial Study Group. Effects of enalapril on mortality in severe congestive heart failure: results of the Cooperative North Scandinavia Enalapril Survival Study (CONSENSUS). N Engl J Med 1987; 316: 1429-35.

3. Keogh AM, Baron DW, Hickie JB. Prognostic guides in patients with idiopathic or ischemic dilated cardiomyopathy assessed for cardiac transplantation. Am J Cardiol 1990; 65: 903-8.

4. Komajda M, Jais JP, Reeve F, et al. Factors predicting mortality in idiopathic dilated cardiomyopathy. Eur Heart J 1990; 11: 824-31.

5. Kelly TL, Cremo R, Nielsen C, et al. Prediction of outcome in late-stage cardiomyopathy. Am Heart J 1990; 119: 1111-21.

6. Koide T, Akihito K, Yutaka T, et al. Variable prognosis in congestive cardiomyopathy: role of left ventricular function, alcoholism, and pulmonary thrombosis. Jap Heart J 1980; 21, 4: 451-63.

7. Stelken AM, Younis LT, Jennison SH, et al. Prognostic value of cardiopulmonartexercise testing using achieved of predicted peak oxygen uptake for patients with ischemic and dilated cardiomyopathy. J Am Coll Cardiol 1996; 27: 345-52.

8. Sparrow J, Parameshwar J, Poole-Wilson PA. Assessment of functional capacity in chronic heart failure: time limited exercise on self-powerer treadmil. Br Heart J 1994; 71:391-4.

9. Williams MJ, Odabashian J, Lauer MS, et al. Prognostic value of dobutamine echocardiography in patients with left ventricular disfunction. J Am Coll Cardiol 1996; 27 : 132-9.

10. Giles TD, Roffidal L, Quiroz A, et al. Circadian variation in blood pressure and heart rate in nonhypertensive congestive heart failure. J Cardiovasc Pharmacol 1996; 28: 733-40.

11. Rosner B. Fundamentals of Biostatistics. $2^{\text {nd }}$ ed. Boston: PWS Publishers, 1988.

12. Baker BJ, Detsky AS, Wesson DE, et al. Predictive value of M-mode echocardiography in patients with congestive heart failure. Am Heart J 1986; 111: 697-702.

13. Gavazzi A, De Maria R, Renosto G, et al. The spectrum of the left ventricular size in dilated cardiomyopathy: clinical correlates and prognostic implications. Am Heart J 1993; 125: 410-22.

14. Mady C, Cardoso RHA, Barretto ACP, et al. Survival and predictors of survival in patients with congestive heart failure due to Chagas cardiomiopathy. Circulation 1994; 90: 3098-102.

15. Ortiz J, et al. One-year mortality prognosis in heart failure: a neural network approached based on echocardiographic data. J Am Coll Cardiol 1995; 26: 1586-93.

16. Ortiz J, Matsumoto AY, Ghefter CGM, et al. Prognosis in dilated myocardial disease: influence of diastolic dysfunction and anatomical changes. Echocardiography 1993; 10: 247-53.

17. Ortiz J, Matsumoto AY, Silva CES. O ecocardiograma na avaliação prognóstica da insuficiência cardíaca. Arq Bras Cardiol 1988; 51: 89-91.

18. Pernenkil R, Vinson JM, Shah AS, et al. Course and prognosis in patients $>70 \mathrm{yrs}$ of age with congestive heart failure and normal versus abnormal left ventricular ejection fraction. Am J Cardiol 1997; 77: 216-9.

19. Marcus RH, Lang RM, Neumann A, et al. A physiological aproach to drug terapy in dilated cardiomyopathy. Echocardiography 1991; 8: 173-86.

20. Devereux RB, Pickering TG. Relationship between the level, pattern and variability of ambulatory blood pressure and target organ damage in hypertension. J Hypertens 1991; 8: S34-8.
21. Rowlands DB, Ireland MA, Glover DR, et al. The relationship between ambulatory blood pressure and echocardiographically assessed left ventricular hypertrophy. Clin Sci 1981; 61: 101-3.

22. Giaconi S, Levanti C, Pommei, et al. Microalbuminuria and casual and ambulatory blood pressure monitoring in normotensives and in patients with borderline and mild essential hypertension. Am J Hypertens 1989; 2: 259-61.

23. Shimada K, Kawamato A, Matsubayashi K, et al. Silent cerebrovascular disease in the elderly: correlation with ambulatory pressure. Hypertension 1990; 16: 692-9.

24. Borne P, van de Abramowicz M, Degre S, et al. Effects of chronic congestive heart failure on 24-hour blood pressure and heart rate patterns: a hemodynamic approach. Am Heart J 1992; 123: 998-1004.

25. Canesin MF, Barretto ACP, Giorgi DM, et al. Ambulatory twenty-four-hours blood pressure variation is associate with cardiac function and quality of life in patients with severe congestive heart failure: follow-up of 12 months. Eur Heart J 1998; 19: 512.

26. Zanchetti A. The role of ambulatory blood pressure monitoring in clinical practice. Am J Hypertens 1997; 10: 1069-80.

27. Verdecchia P, Porcellati C, Schillaci G, et al. Ambulatory blood pressure: an independent predictor of prognosis in essencial hypertension. Hypertension 1994; $24:$ 793-801.

28. Verdecchia P, Schillaci G, Porcellati C. Dippers x non-dippers. J Hypertens 1990; 9: $\mathrm{S} 42-\mathrm{S} 4$

29. Bridgen W, Sharpey-Schafer EP. Postural changes in peripheral blood flow in cases with left heart failure. Clin Sci 1950; 9: 93-100.

30. Zucker IH, Earle AM, Gilmore GP. The Mechanism of adaptation of left atrial stretch receptors in dogs with chronic congestive heart failure. JClin Invest 1997; 60: 323-31.

31. Zucker IH, Earle AM, Gilmore GP. Changes in the sensitivity of left atrial receptors following reversal of heart failure. Am J Physiol 1979; 237: H555-9.

32. Levine TB, Francis GS, Goldsmith SR, et al. Activity of the sympathetic nervous system and renin-angiotensin system assessed by plasma hormone levels and their relationship to hemodynamic abnormalities in congestive heart failure. Am J Cardiol 1982; 49: 1659-66.

33. Imai Y, Aihara A, Ohkubo T, et al. Factors that affect blood pressure varibility. Am J Hyperten 1997; 10: 1281-9.

34. Parati G, Pomidossi G, Albini F, et al. Relationship of 24 hour blood pressure mean and variability to severity of target-organ damage in hypertension. J Hypertens 1987; 5: 93-8.

35. Schillaci G, Verdecchia P, Borgioni C, et al. Lack of association between blood pressure variability and left ventricular mass in essential hypertension. Am J Hyperten 1988; 11: 515-22.

36. Caruana MP, Lahiri A, Cashman PMM, et al. Effects of chronic congestive heart failure secondary to coronary artery disease on the circadian rhythm of blood pressure and heart rate. Am J Cardiol 1988; 62: 755-9.

37. Franciosa JA, Wilen M, Ziesche S, et al. Survival in men with severe chronic left ventricular failure due to either coronary artery disease or idiopathic dilated cardiomyopathy. Am J Cardiol 1983; 51: 831-6.

38. Cleland JGF, Dargie HJ, Ford I. Mortality in heart failure: clinical variables of prognostic value. Br Heart J 1987; 58: 572-82.

39. Ghali JK, Kadakia S, Bhatti A, et al. Survival of heart failure patients with preserved versus impaired systolic function: the prognostic implication of blood pressure. Am Heart J 1992; 123: 993-7. 